\title{
CRISPR system for genome engineering: the application for autophagy study
}

\author{
Jianzhou Cui ${ }^{1, *}$, Shirley Jia Li Chew ${ }^{1}$, Yin Shi ${ }^{1}$, Zhiyuan Gong $^{2}$ \& Han-Ming Shen ${ }^{1,3, *}$ \\ ${ }^{1}$ Department of Physiology, Yong Loo Lin School of Medicine, National University of Singapore, ${ }^{2}$ Department of Biological Sciences, \\ National University of Singapore, ${ }^{3}$ NUS Graduate School for Integrative Sciences and Engineering, National University of Singapore, \\ Singapore
}

CRISPR/Cas9 is the latest tool introduced in the field of genome engineering and is so far the best genome-editing tool as compared to its precedents such as, meganucleases, zinc finger nucleases (ZFNs) and transcription activator-like effectors (TALENs). The simple design and assembly of the CRISPR/Cas9 system makes genome editing easy to perform as it uses small guide RNAs that correspond to their DNA targets for high efficiency editing. This has helped open the doors for multiplexible genome targeting in many species that were intractable using old genetic perturbation techniques. Currently, The CRISPR system is revolutionizing the way biological researches are conducted and paves a bright future not only in research but also in medicine and biotechnology. In this review, we evaluated the history, types and structure, the mechanism of action of CRISPR/Cas System. In particular, we focused on the application of this powerful tool in autophagy research. [BMB Reports 2017; 50(5): 247-256]

\section{INTRODUCTION}

Genome engineering technology has come a long way since its humble beginning in the 1970s and since then, it has undergone rapid development which saw better, more efficient and robust tools for use in genetic perturbations. Genome engineering is essentially the process of modifying the genetic configuration of an organism in a targeted and specific manner, and encompasses the strategies or techniques to carry out the modification process as well. Such a breakthrough in biology has permitted researchers to expand our knowledge of what is known about gene function and the

*Corresponding authors. Jianzhou Cui, Tel: +65-6516-6406; Fax: +65-6779-1489; E-mail: csicuij@nus.edu.sg, Han-Ming Shen, Tel: +65-6516-4998; Fax: +65-6779-1489; E-mail: han-ming_shen@ nuhs.edu.sg

https://doi.org/10.5483/BMBRep.2017.50.5.044

Received 13 March 2017

Keywords: Autophagy, CRISPR/Cas9, gRNA, Off-target, PAM capacity to alter DNA also allows researchers to model human diseases in animal models, making it possible to exploit this for gene therapy and drug development (1).

To date, there are currently four major classes of genome editing technologies namely meganucleases, zinc finger nucleases (ZFNs), transcription activator-like effectors (TALENs) and the most recent addition, the clustered regularly interspaced short palindromic repeats (CRISPR) and CRISPRassociated (Cas) (CRISPR/Cas) as well as the CRISPR-Cpf1 (CRISPR from Prevotella and Francisella 1) systems (2, 3). These four technologies manipulate genetic material by inducing site-specific DNA double-strand breaks (DSBs) that result in genome editing either via homologous recombination (HR)-mediated recombination events or non-homologous end joining (NHEJ) (4). Although all of them are collectively classified under the same category of programmable nucleases, the mechanism of each of these genome editing technologies differ from one another.

In general, meganucleases, ZFNs and TALENs nucleases target specific DNA sequences through protein-DNA interactions (5). Meganucleases, also known as homing endonucleases, are nature's highly specific nucleases whereby its nuclease and DNA-binding domains are combined into one single domain. In contrast, ZFNs and TALENs are artificially engineered nucleases with a DNA binding domain fused to a non-specific nuclease domain of Fokl. In this sense, meganucleases are not as efficient as ZFNs and TALENs because they are limited in their capacity to bind to new DNA sequences with high specificity. ZFNs and TALENs would seemingly be better alternatives but these two tools are not without drawbacks. The complication of context-dependent binding preference between individual finger domains of ZFNs make designing of programmable ZFNs difficult even though solutions have been drawn up to address this limitation (6) as extensive screening process is necessary. On the other hand, TALENs exhibit lesser context-dependent binding preference and their modular assembly makes it possible to target any DNA sequence (7). Furthermore, assembly of DNA encoding the repetitive domains of TALENs requires unconventional molecular biology cloning methods which can be costly in terms of time and labor (8). Now, genome engineering technology has seen

ISSN: 1976-670X (electronic edition)

Copyright (c) 2017 by the The Korean Society for Biochemistry and Molecular Biology

(c) This is an open-access article distributed under the terms of the Creative Commons Attribution Non-Commercial License (http://creativecommons.org/licenses/by-nc/4.0) which permits unrestricted non-commercial use, distribution, and reproduction in any medium, provided the original work is properly cited. 
widely used with the advent of the CRISPR system that has shown promising results in addressing the issues pertinent to modular DNA-binding protein construction. The CRISPR system has been employed in a variety of studies for its ease of customization to target any desired DNA sequences in a genome simply via customized sgRNA (4).

Autophagy is an evolutionarily conserved pathway for degradation of cytoplasmic proteins and organelles via lysosome. Proteins coded by the autophagy-related genes (Atgs) are the core molecular machinery in control of autophagy. The ability of precise genome editing of autophagyrelated-genes (Atgs) plays a critical role to study the underlying mechanisms of this complex process (9). The first knockout Atgs is Beclin1 via embryonic stem (ES) cell-based genetargeting technique in mice, which showed significant phenotypes $(10,11)$. After that, many Atgs such as $A T C 4 B$, ATG5, ATG7 have been modified in cells and mice using Cre-Lox recombinase and/or ES cell-based gene-targeting approaches to study the role of those Atgs in autophagy regulation and related biological functions (12-16). Recently, the CRISPR system have been developed and the convenience of design, construction, and delivery of sgRNAs offered an excellent possibility of rapid genome editing in autophagy study via targeting Atgs using CRISPR system. Here, we reviewed the very recent study of the novel genome editing tool CRISPR in knockout of autophagy genes and reported our partial date in order to elaborate the important role of CRISPR in autophagy research.

\section{THE CRISPR STORY}

\section{History of CRISPR}

CRISPR systems have created a profound and lasting effect ever since it was established as the latest genome editing tool along with past technologies like meganucleases, ZFNs and TALENs. CRISPR clustered repeats was first discovered in 1987 while Nakata and team were working on the IAP enzyme in $E$. coli and a set of 29-nt repeats downstream of the iap gene was found (17). In 2002, Jansen and Mojica collectively described the genomic loci of microbials which consists of an interspaced repeat array with the term CRISPR (18). The research on CRISPR was at its crux in 2005 when further analyse on spacer sequences that separate each direct repeats resulted in the conclusion that they were of extra chromosomal and phage-related nature (19).

By 2010, the functional mechanism of the natural Type II CRISPR system was better understood to construct an RNA-guided DNA endonuclease for genome editing. Cas9 is the sole enzyme within the cas gene array to exert nucleolytic activity on DNA (20). Together with this data, a non-coding trans-activating crRNA (tracrRNA) which hybridizes with crRNA to facilitate RNA-guided targeting of Cas9 has been to be the key component in crRNA biogenesis and processing in Type II CRISPR system (21). Later in 2012, it was shown that crRNA-guided cleavage by purified Cas9 was possible (22) and that a single guide RNA (sgRNA) could be designed by joining a crRNA containing the guide sequence to a tracrRNA (23) which aids DNA cleavage by Cas9. Currently, multiple guide RNAs could also be designed to target multiple genes at once for genome editing with high efficiency (24). Many opensource distributors and online user forums have helped to advance the Cas 9 technology as well.

\section{Types of CRISPR/Cas system}

The CRISPR adaptive immune system and CRISPR-associated (Cas) systems which originated from bacterial and archaeal hosts (25) primarily function as an immune system that cleaves exogenous DNA (26) or RNA (27) via an RNA-guided nuclease. Therefore, this serves to protect the bacterial and archaeal hosts from invading viruses or plasmids. CRISPR systems have been classified into three major types (Table 1) based on their genetic content, structural and functional differences whereby the key differences among the three is established by the Cas genes and encode proteins (28). However, among the three, the Type II CRISPR system is the best characterized (29) which comprises of the nuclease Cas9, the crRNA array and an ancillary trans-activating crRNA (tracrRNA).

\section{The structure of Cas9}

It was found that S. pyogenes Cas9 (SpCas9 for short) has two lobes; recognition (REC) lobe and nuclease (NUC) lobe (30).

Table 1. 3 Major types of CRISPR systems

\begin{tabular}{|c|c|c|c|c|c|c|c|c|c|c|c|}
\hline \multirow{2}{*}{$\begin{array}{c}\text { Types } \\
\text { Subtypes }\end{array}$} & \multicolumn{5}{|c|}{ I } & \multicolumn{4}{|c|}{ II } & \multicolumn{2}{|c|}{ III } \\
\hline & A & B & $\mathrm{C}$ & $\mathrm{D}$ & $\mathrm{E}$ & $\mathrm{F}$ & A & B & $\mathrm{C}$ & A & B \\
\hline Organism & \multicolumn{5}{|c|}{ Bacteria } & \multicolumn{4}{|c|}{ Bacteria } & \multicolumn{2}{|c|}{ Archaea \& Hyperthermophiles } \\
\hline Target & DNA & & & & & DNA & & & & DNA & RNA \\
\hline Genetic & \multicolumn{5}{|c|}{ cas $1, \operatorname{cas} 2, \operatorname{cas} 3 *, \operatorname{cas} 5, \operatorname{cas} 6, \operatorname{cas} 7$} & \multicolumn{4}{|c|}{ cas $1, \operatorname{cas} 2, \operatorname{cas} 9 *$} & \multicolumn{2}{|c|}{$\operatorname{cas} 1, \operatorname{cas} 2, \operatorname{cas} 6, \operatorname{cas} 10^{*}$} \\
\hline $\begin{array}{l}\text { Signature genes } \\
\text { References }\end{array}$ & $\begin{array}{l}\text { cas8a } \\
(28)\end{array}$ & cas $8 b$ & $\operatorname{cas} 8 \mathrm{c}$ & cas10d & cse1, cse 2 & $\begin{array}{l}\text { Csy } 1 \\
(28)\end{array}$ & $\operatorname{csn} 2$ & cas4 & - & $\begin{array}{l}\operatorname{csm} 2 \\
(78)\end{array}$ & cmr5 \\
\hline
\end{tabular}


The variable, $\alpha$-helical REC lobe is composed of three regions as follows: a long $\alpha$ helix referred to as the bridge helix, REC1 domain and REC2 domain (31). The NUC lobe similarly has three domains but they are the RuvC, $\mathrm{HNH}$ and PAMinteracting (PI) domains. Within both of these two lobes, two clefts that bind to gRNAs and target DNA sequences by the REC and NUC lobes respectively were identified. Between the REC and NUC lobes is a positively charged groove, which is formed as a result of RuvC domain interfacing with PI domain, where the negatively charged sgRNA:target DNA heteroduplexdocks.

As aforementioned, Cas9 must first undergo conformational change to activate its catalytic function. Based on singleparticle electron microscopy reconstructions, conformation of Cas9 in the apo (unbound) state do not permit binding and cleaving of target DNA (32). It is only upon association of crRNA-tracrRNA duplex with Cas9 that it induces the two lobes to rearrange its structure into a channel for the target sequence to dock (33). Hence, the presence of the crRNAtracrRNA duplex determines if Cas9 is activated or not. Additionally, the $\mathrm{HNH}$ and RuvC domains can be mutated for functions other than for carrying out strand-specific cleavage (29). By substituting aspartate with alanine (D10A) in the RuvC domain, the mutant Cas9 now nicks DNA to yield singlestranded breaks and the favored homology-directed repair (HDR) potentially reduces the frequency of undesirable indels from off-target DSBs (29).

With a thorough and deeper understanding of the mechanism for the Type II CRISPR/Cas system, attempts to redesign its structure to facilitate genome engineering purpose have been successful. The result was the construction of a chimeric RNA with crRNA and tracrRNA-derived sequences which was subsequently named as guide RNA (gRNA) (23). For highly specific DNA targeting, the crRNA or gRNA can be redesigned to target any DNA sequences and guide Cas9 to result in sequence-specific DSB.

\footnotetext{
Mechanism of action

The Type II CRISPR/Cas system derived from the SpCas9 has been studied the most extensively and as such, its mechanism of action is one of the most established (34). One crRNA unit consists of a partial direct repeat and a 20-nt guide sequence that is responsible for guiding Cas9 to a complementary 20-bp DNA target via Watson-Crick base pairing. The crRNA and tracrRNA fuse together to form a two-RNA structure which binds to either strand beside a PAM sequence. The target DNA precedes a 5'-NGG PAM (23) that is important for target recognition of Cas9 nuclease. This double-stranded (ds) DNA endonuclease targets specific sites for cleavage via crRNA and tracrRNA to stimulate a DSB.

Through single-molecule imaging, Cas9-gRNA complex was observed to strongly interact with target sequence containing a PAM (35) as compared to non-target sequences or complementary sequences lacking PAMs whereby binding was
}

observed to be transient. Following PAM recognition, the Cas9-gRNA must unwind the double helix and initiate strand separation for complementary base pairing to occur between the target DNA and the crRNA guide sequence. It has been postulated (35) that PAM binding could either cause a destabilization of the DNA duplex along the length of the target sequence leading to random nucleation of the RNADNA heteroduplex or cause a local melting of the duplex. The latter involves the RNA-DNA heteroduplex nucleating at the $3^{\prime}$ end that is adjacent to PAM before nucleating towards the $5^{\prime}$ end.

In addition to SpCas9, more than 20 additional Cas9 homologs derived from a variety of bacterial species have been isolated. The PAM sequences also shows the big variation which can provide more option to us when no suitable SpCas9 PAM available in the gene you interested (36).

\section{A Cas9 homolog: Cpf1}

Cpf1, a putative new class 2 nuclease was recently annotated from Feng Zhang's lab. Cpf1 is classified as a novel, type V CRISPR system. Cpf1 contains a RuvC-like endonuclease domain which is similar to Cas9, but without $\mathrm{HNH}$ endonuclease domain, indicating that Cpf1 may shows different function $(3,37)$.

Cpf1 cleaves DNA requires only one RNA rather than the two (tracrRNA and crRNA) which is more convenient than Cas9. In addition, Cpf1's preferred PAM is $5^{\prime}-\mathrm{TTN}$, differing from that of Cas9 (3'-NGG) in both genomic location and GC-content. In terms of cleavage pattern, Cpf1 can cause 5 nucleotide $5^{\prime}$ overhang which is also different to Cas9 that created blunt double stranded cleavage (38). Since both Cpf1 and its guide RNAs are smaller than those in the SpCas9 system, they will also be easier to deliver in low-capacity vectors and shows high efficiency. Hence, the introduction of Cpf1-driven systems has added another option to the CRISPR toolbox and the application of Cpf1 to genome editing shows the potential advantages over Cas9 system.

\section{APPLICATIONS AND DELIVERY SYSTEM}

\section{Native Cas9-mediated genome editing}

As stated earlier, the native Cas9-mediated genome editing is executed through two steps. Firstly, Cas9 induces a DSB at a targeted site on the genomic DNA which is guided by a 20-nt guide sequence in the crRNA. Secondly, the DSBs then undergo either the error-prone NHEJ or the high-fidelity HDR pathway. For the native Cas9 system to work, the basic components required includes the Cas 9 nuclease, tracrRNA and the customizable crRNA which should all be expressed in the foreign host. With the ease of customization of the 20-nt guide sequences, double deletion and/or multiplexed editing were made possible in E. coli (39) and human (40) genomes in one step.

When the Type II CRISPR/Cas system was further simplified 
to include just the Cas9 nuclease and custom gRNAs, it opened up an even broader selection of cell types and organisms for genome editing. Studies to date have successfully engineered and edited the genomes of humans, mice, fruit flies, zebrafish, yeast, thale cress, tobacco, wheat and rice plants (41). This simplified version of the Type II CRISPR/Cas system was observed to be capable of disrupting five genes in a single genome simultaneously (42). Indeed, the Type II CRISPR/Cas system is an excellent platform for genomic studies with broad applications in a variety of hosts.

\section{Cas9 nickase-mediated genome editing}

By mutating the RuvC or $\mathrm{HNH}$ domain, the gRNA-guided Cas9 which originally induces a DSB at the target site now has the acquired nickase ability. This mutated complex, gRNAguided Cas9n, with nicking function is useful for successful genome editing at target sites specifically through the generation of DSBs and NHEJ-induced mutations when used as a pair (41). This double nicking strategy targets the opposite strand of a target site to initiate HDR that is higher in efficiency and faster in rate as compared to the native Cas9-mediated HDR and single Cas9n-mediated HDR respectively (43).

Off-target cleavages were surprisingly reduced by 50 to 1500 times in human cells via this paired nicking mechanism without comprising the efficiency of on-target cleavages (43). One other advantage brought about by paired nicking is that it generates accurate overhangs as predicted. Together with NHEJ-mediated ligation, double-stranded repair templates with complementary overhangs have demonstrated success in HDR-independent fragment integration at target sites (41). Furthermore, about $6 \mathrm{~kb}$ worth of genomic fragments in HEK293FT cells were deleted when paired double nicks are induced at two sites using four customized gRNAs. As such, it could be concluded that Cas9n is able to induce highly accurate genome editing.

\section{Inactivated Cas9-based transcriptional control}

A completely inactive dCas9 coupled with a custom gRNA is able to exert transcriptional control without changing the target sequence. Such inactivated Cas9-based transcriptional control is termed as CRISPR interference (CRISPRi) and identifies target sequences via complementary base pairing. Once the target sequence is identified, CRISPRi inhibits the initiation of transcription and elongation (41) which were successfully observed in E. coli and human cells (44). Since the gRNAs are customizable, it makes it plausible to exploit this system for regulating several genes at any one time.

Besides inhibiting the initiation of transcription and elongation, genes could also be silenced and this effect is made reversible with anhydrotetracycline-inducible promoter to initiate dCas9 and gRNA expression (44). The efficiency of this system to repress transcription was found to attain repression about 1000 fold (44) which proves its usefulness in gene expression regulation at the transcriptional level.

\section{Gene therapy}

Besides its use in genetic studies in many species, Cas9 can be utilized to model the causal roles of specific genetic variations in human induced pluripotent stem cells (iPSCs) with specific mutations introduced or rectified (45). Human iPSCs are useful as they are a renewable source of cells for human biology and disease research and are also a potential candidate for gene or cell therapy development (46). In one recent study conducted by Smith et al., whole-genome sequencing analysis was performed and it was found that CRISPR/Cas9 demonstrated high specificity genome editing in human iPSCs. However, it remains to be evaluated in this study if the higher off-target rates observed in cancer cell lines are accounted by gRNAs and Cas9 overexpression and/or due to aggravated faulty repair systems in these cells.

Currently, ongoing studies are being conducted on the therapeutic potentials of the CRISPR/Cas9 system and results have been promising (47). Non-genetic or genetic disorders, which is largely due to point mutations, substitutions, deletions and insertions (48), or complex diseases could be rectified with engineered endonuclease Cas9. In the search for a form of cure for AIDS, it was understood that individuals who are homozygous for $\Delta 32$ deletion in CCR5 (CCR5 $\Delta 32$ ) have resistance to CCR5-tropic HIV-1 infections (49). Therefore, it is highly possible that through specific deletions executed by the engineered endonuclease Cas9, this novel technology could be a gateway for an eventual cure for AIDS (50). Moreover, besides a feasible strategy to circumvent HIV infection, deletion of PCSK9 (50) or angiopoietin (51) have demonstrated convincing results as a potential means against stain-resistant hypercholesterolemia or hyperlipidemia.

\section{The different delivery systems of CRISPR}

The CRISPR system can be delivered into a wide range of cell types and organisms (Table 2) as mentioned above through various delivery techniques. Electroporation (52), nucleofection and Lipofectamine-mediated transfection (53) of non-replicating plasmid DNA have been utilized to transiently express Cas9 and gRNAs in mammalian cells in vitro (54-56). These methods are traditionally used to deliver RNA-guided nucleases but in terms of efficiency, it is not as efficient as lentiviral vectors which confer very high gene delivery efficiency of about $95-100 \%$.

On the other hand, it is also possible to directly introduce RNAs and plasmid DNA by microinjection into zebrafish, fruit flies, mice and rats embryos (54). Gonads of roundworms were similarly subjected to the same direct injection method in addition to the direct injection of purified Cas9 protein complexed with gRNA (57) in a separate study. Not only in animals, Cas9 was also successfully delivered into many plant species such as wheat, rice, sorghum, tobacco and thale cress via delivery methods like PEG-mediated transformation of protoplasts, Agrobacterium-mediated transfer in embryos and leaf tissue and/or bombardment of callus cells with plasmid 
Table 2. Application of Type II CRISPR/Cas system in various cell types and organisms

\begin{tabular}{|c|c|c|}
\hline Application & Cell types/Organisms & References \\
\hline \multirow{16}{*}{$\begin{array}{r}\text { Genome } \\
\text { editing }\end{array}$} & Mouse & $(40)$ \\
\hline & $\begin{array}{l}\text { Human HUES62, HEK293T, 293FT, } \\
\text { K562 \& iPS cells }\end{array}$ & $(40,77-79)$ \\
\hline & Rice protoplast and callus cells & $(80-83)$ \\
\hline & Streptococcus pneumoniae & (39) \\
\hline & Escherichia coli & (39) \\
\hline & Zebrafish embryos & $(79,84)$ \\
\hline & Drosophila preblastoderm embryos & $(85)$ \\
\hline & Caenorhabditis elegans germ line & $(86,87)$ \\
\hline & Xenopus tropicalis embryos & (88) \\
\hline & Saccharomyces cerevisiae & (89) \\
\hline & Arabidopsis protoplast and seedlings & $(39,90)$ \\
\hline & Wheat protoplast & (83) \\
\hline & Tobacco protoplast and leaf & $(81,90,91)$ \\
\hline & Sorghum immature embryos & (81) \\
\hline & C. elegans germ line & $(92)$ \\
\hline & Mouse zygotes & $(43)$ \\
\hline \multirow{4}{*}{$\begin{array}{l}\text { Transcriptional } \\
\text { control }\end{array}$} & E. coli & (93) \\
\hline & S. pneumoniae & (93) \\
\hline & Human HEK293, 293T cells & $(77,94)$ \\
\hline & S. cerevisiae & $(94)$ \\
\hline
\end{tabular}

DNA (58).

\section{Off-target effects}

The issue of off-targeting exists because CRISPR/Cas9 tolerates mismatches up to 5-bp within the protospacer region (53). When the gRNA binds to a site within the genome that is not completely complementary, the Cas9 nuclease is misguided to stimulate a DSB at the off-target site instead. The repercussion of off-target cleavage would be a mis-interpretation of phenotypic effects in gene knockout experiments which could also result in undesirable toxicities (59). Off-targeting is positively correlated with Cas9 concentration whereby off-target activity becomes more significant as concentration of Cas9 increases (60). However, this problem could be easily solved by adjusting Cas 9 concentration and hence, the Cas9-sgRNA complex levels. As evident in the study by $\mathrm{Hsu}$ et al., specificity increased significantly as equimolar amounts of Cas9 and sgRNA transfected into 293FT cells were reduced from $400 \mathrm{ng}$ to $10 \mathrm{ng}$ of Cas9-sgRNA plasmid (60). Besides regulating Cas9-sgRNA complex levels, decreasing the amount of transfected DNA was another method to increase specificity (60). Although effective, decreasing the amount of transfected DNA would have an effect on on-target cleavage.

Facing such an issue, two independently discovered CRISPR variants: eSpCas9 and SpCas9-HF1 have been development to improve the on-target specificity respectively (61). Slaymaker et al believed that, if they decreased the positive charge in the $\mathrm{HNH} /$ RuvC groove would theoretically decrease off-target cutting (61). Hence, a variety of alanine substitutions throughout the groove in 32 separate Cas9 mutants had created to decrease the electropositivity of the $\mathrm{HNH} /$ RuvC groove. Two of the mutants, SpCas9 (K855A) and eSpCas9, revealed that these mutants do not cause off-target effects at unanticipated sites. Meanwhile, Kleinstiver et al. reasoned that the weaken sequence independent interactions between Cas9 and DNA also could diminish off-target cutting (62). SpCas9-HF1 (mutation Q926A) generated fewer off-target cuts when compared to WT SpCas9 across a variety of genomic sites. Accordingly, with the enhanced specificity or other mutation combination, eSpCas9 and SpCas9-HF1 should enable researchers to make precise edits in mammalian cells with decrease worries about off target effects in future.

\section{CRISPR AS A POWERFUL TOOL IN AUTOPHAGY STUDY}

\section{Current application of CRISPR in autophagy}

Although many chemical inhibitors of autophagy had been widely used in autophagy study, those can block a given pathway but have limitations for specific and efficient inhibition. For example, some inhibitors such as chloroquine, bafilomycin A1, and 3-methyladenine (3-MA), can target many components or many aspects of the autophagic pathway, suggesting that these inhibitors are not exclusive (16). Chloroquine and bafilomycin A1 can block autophagy by impairing lysosomal function, while 3-MA is an inhibitor of phosphoinositide 3-kinase (PI3K) $(63,64)$. In addition, these inhibitors also play an important role in other pathways involved in other different physiology function $(65,66)$.

To enhance the specification of the inhibition of autophagy, siRNA and shRNA had been used to inhibit autophagy by genetic silencing of ATG genes. Compared with those pharmacological inhibitors, these strategies show more specific function in autophagy inhibition. While the knockdown effects caused by gene silence are often incomplete so as to the autophagy inhibition. Hence we need new genome-engineering strategies, such as CRISPR/Cas9 to achieve complete gene deletion and autophagy inhibition.

With the development of the excited CRISPR technique, researchers had applied this tool to autophagy research field. Since autophagy is an evolutionarily conserved pathway and proteins coded by the autophagy-related genes (Atgs) are the core molecular machinery in control of autophagy (9). The ability of precise genome editing of Atgs plays a critical role to study the underlying mechanisms of this complex process (Fig. 1). Currently, most works for genome editing of the Atgs were focued on the gene knockout as well as knockin (67). The effects of several Atg genes knockout have been well studied from the formation of autophagosomes to autolysosomal biogenesis $(68,69)$. Using CRISPR/Cas9 to delete of the 


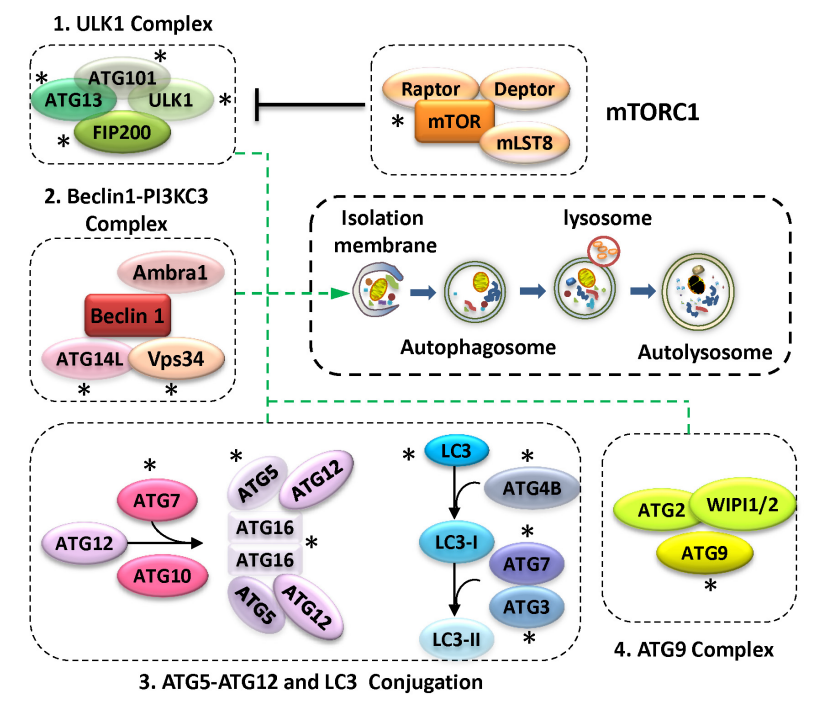

Fig. 1. Schematic diagram of the mammalian autophagy core machinery and CRISPR/Cas9 targeted genes. The Atg proteins form several important functional groups in control of autophagosome formation. (i) The ULK1 complex, consisting of the serine/threonine kinase ULK1, ATG13, focal adhesion kinase family interacting protein of $200 \mathrm{kDa}$ (FIP200) and ATG101, controls the induction or initiation of autophagy for the formation of phagophore and is negatively regulated by mechanistic target of rapamycin (mTOR). (ii) The Beclin 1-class III PI3K complex controls the nucleation step of autophagosome formation. (iii) The two ubiquitin-like conjugation systems (the ATG12-ATG5 system and the LC3 system) mediate the elongation stage, leading to formation of a complete autophagosome. And (iv) The ATG9 retrieval process functionally involves a protein complex of WIPI1/2 and ATG2. The CRISPR targeted genes (Table 3 ) in the autophagy core machinery in this review was indicated by an asterisk $(*)$. canonical autophagy-essential genes ATG5, ATG7, ATG16L and ULK1 have also been reported in in vitro or in vivo models (70-74). In addition, A genome-wide CRISPR screen in MTOR signaling and the entire macroautophagy machinery as key regulators of SQSTM1 suggesting that the pooled CRISPR screening as a powerful method to map the cellular pathways that regulate the fate of an individual target protein (75) (Table 3). Table 3 summarized the recent studies of the CRISPR/Cas9 application for Atgs target. Next, we present our own data on ATG7 knockout and investigate the effect of ATG7 deletion on autophagy.

\section{ATG7 knockout blocks basal and starvation-induced autophagy level}

Here, two construct systems pSpCas9 (BB)-2A-GFP (PX458) (Addgene plasmid \#48138) and pSpCas9 (BB)-2A-Puro (PX459) (Addgene plasmid \#48139) were used to investigate the effect of ATG7 knockout on autophagy. Sanger sequencing results show that in instances where indels, do occur, they were mostly found within the target sequences. Overall, indel rates for the two target sites of ATG7 (ATG7-1 and ATG7-2) in both PX458 and PX459 transfection systems were $87.0 \%$, $75.0 \%$ and $85.0 \%, 84.7 \%$ respectively (Data not shown).

ATG7 protein levels were significantly lower in knockout mutants as compared to the controls, non-transfected cells and cells transfected with empty vectors. There is a general trend of high SQSTM1 protein levels among knockout mutants as observed. It was also noted that mutants had lower LC3-II protein levels than the controls suggesting that the ATG7 knockout effectively blocks the basal level of autophagy in the two Cas9 systems (Fig. 2A).

Under starvation condition, ATG7 mutants generally had relatively higher levels of SQSTM1 and lower levels of LC3-II

Table 3. The genes targeted by CRISPR/Cas9 involved in autophagy core machinery

\begin{tabular}{|c|c|c|c|}
\hline Target Genes & Effects on autophagy & Delivery systems & Refs \\
\hline ULK1, ATG101 & Suppression of induction & Transfected into U937, MEFs & $(70,95)$ \\
\hline ATG5 & $\begin{array}{l}\text { Resistance to gossypol in ATG5 knockout cells is } \\
\text { associated with increased cytoprotective autophagy, } \\
\text { independent of ATG5. }\end{array}$ & $\begin{array}{l}\text { Transfected into A375P cells, } \\
\text { IPEC-J2. }\end{array}$ & $(72,96)$ \\
\hline ATG3, ATG7, ATG13 & $\begin{array}{l}\text { Suppressed translation and ULK1 degradation can restrict } \\
\text { autophagy under prolonged starvation; Suppression of } \\
\text { Vesicle Elongation }\end{array}$ & $\begin{array}{l}\text { lentiCRISPR v1 vector; } \\
\text { Transfection in K562; }\end{array}$ & $(71,73,97-99)$ \\
\hline Atg8/LC3 & $\begin{array}{l}\text { Knockout of LC3/GABARAPs was failure to drive } \\
\text { autophagosome-lysosome fusion }\end{array}$ & Transfected into HeLa cells & (69) \\
\hline ATG9 & Suppression of retrieval process & Injecting an expression plasmid & $(100)$ \\
\hline ATG16L1 & Suppression of Vesicle Elongation & Transfected into HAP1 cells & (74) \\
\hline SQSTM1/MTOR & $\begin{array}{l}\text { Pooled CRISPR screening to map MTOR signalling and } \\
\text { the entire macroautophagy machinery }\end{array}$ & lentiviral delivery into $\mathrm{H} 4$ cells & (75) \\
\hline VPS34/ATG14 & Suppression of vesicle nucleation & Transfected into HEK293 & $(101,102)$ \\
\hline SMPD1 & $\begin{array}{l}\text { Induces a severe autophagy defect characterized by } \\
\text { altered trafficking of ATG9A }\end{array}$ & Transfected into MCF7 & $(103)$ \\
\hline
\end{tabular}


A

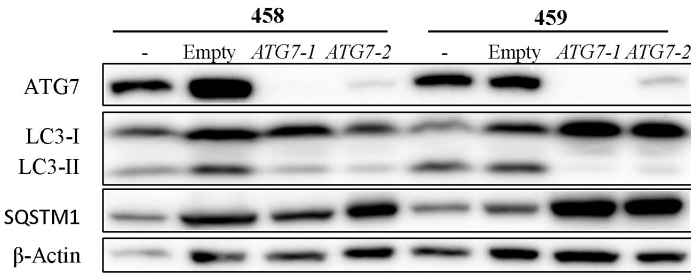

B

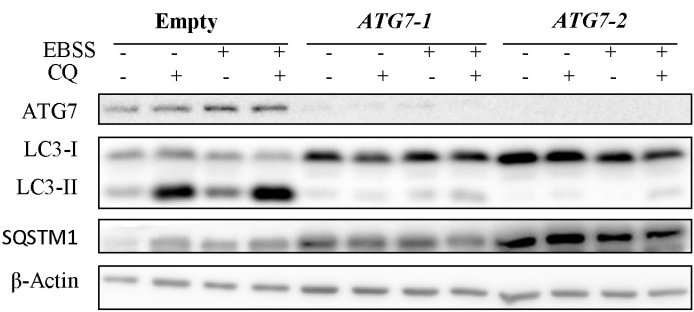

Fig. 2. The effect of knocking out ATG7 on autophagy. (A) The effect of knocking out ATG7 on basal level of autophagy in the two Cas9 systems. (B) The effect of starvation and/or CQ treatment on autophagic flux in ATG7 knockout cells in PX458 systems. Immunoblotting of ATG7, LC3 and SQSTM1 using lysates from HEK293FT cells transfected with respective vectors inserted with two target sites, either ATG7-1 or ATG7-2. $\beta$-ACTIN served as a loading control. Cells were subjected to EBSS starvation and/or $50 \mu \mathrm{M}$ of CQ treatment for two hours.

than the control cells transfected with empty vectors regardless EBBS (Earle's Balanced Salt Solution) and/or CQ treatment. As expected in PX458 transfection systems (Fig. 2B), when controls in both transfection systems were administered EBSS and CQ simultaneously, levels of LC3-II in mutant were significantly reduced compare to control. Thus, we believe that the disruption of ATG7 genes induced by CRISPR-Cas9 either at single or multiple target sites could effectively reduce the basal and starvation induced autophagy level. In addition, ATG7-1 and ATG7-2 knockout mutants exhibited significantly greater cell death under starvation conditions (EBSS treatment groups) as compared to mutants that were not starved (DMEM treatment groups) suggesting that ATG7 knockout promotes cell death (data not shown).

\section{CONCLUSION}

Clearly, the understanding of the CRISPR/Cas9 system has been evolving over the past 3 decades and so far, the system has proved itself to be full of potential in the field of genome engineering. The customizable 20-nt guide sequence of the Cas9-gRNA complex confers flexibility to the CRISPR/Cas9 system to recognize any desired target sequences. The simplification of the system through the construction of a chimeric RNA to give rise to the gRNA has allowed greater targeting efficiency and multiplexible genome targeting. Although primarily found to provide immunity against exogenous genetic elements, the CRISPR/Cas9 system was found to be involved in various other applications as well as in autophagy study. Since this system is still evolving, we believe that in the future we will be able to understand the mechanism behind CRISPR systems better and be well equipped to operate this technology more cost effectively. The CRISPR/Cas9 system has aided in many genomic studies in many areas where traditional tools were unable to achieve. This emerging trend of genome editing is set to potentially help to study the underlying mechanisms in autophagy research in detail via genome modification. Since CRISPR-Cas9 system is an appealing approach to disrupt specific genomic regions with the easily designed gRNA. This rapid and convenient technique might facilitate understanding of molecular mechanisms of a wide range of biological function and regulation involving in autophagy process.

\section{ACKNOWLEDGEMENTS}

This work is supported by research grants from Singapore National Medical Research Council (NMRC/CIRG/1346/2012) to HMS and ZYG.

\section{CONFLICTS OF INTEREST}

The authors have no conflicting financial interests.

\section{REFERENCES}

1. Geurts AM, Cost GJ, Freyvert Y et al (2009) Knockout rats via embryo microinjection of zinc-finger nucleases. Science 325, 433

2. Mali P, Yang L, Esvelt KM et al (2013) RNA-guided human genome engineering via Cas9. Science 339, 823-826

3. Zetsche B, Gootenberg JS, Abudayyeh OO et al (2015) Cpf1 is a single RNA-guided endonuclease of a class 2 CRISPR-Cas system. Cell 163, 759-771

4. Niu JW, Zhang B and Chen H (2014) Applications of TALENs and CRISPR/Cas9 in Human Cells and Their Potentials for Gene Therapy. Mol Biotechnol 56, 681-688

5. Stranneheim $\mathrm{H}$ and Lundeberg J (2012) Stepping stones in DNA sequencing. Biotechnol J 7, 1063-1073

6. Sander JD, Dahlborg EJ, Goodwin MJ et al (2011) Selection-free zinc-finger-nuclease engineering by contextdependent assembly (CoDA). Nat Methods 8, 67-69

7. Maeder ML, Angstman JF, Richardson ME et al (2013) Targeted DNA demethylation and activation of endogenous genes using programmable TALE-TET1 fusion proteins. Nat Biotechnol 31, 1137-1142

8. Uhde-Stone C, Gor N, Chin T, Huang J and Lu B (2013) A do-it-yourself protocol for simple transcription activator-like effector assembly. Biol Proced Online 15, 3

9. Mizushima N (2007) Autophagy: process and function. Genes Dev 21, 2861-2873

10. Qu X, Yu J, Bhagat G et al (2003) Promotion of tumorigenesis by heterozygous disruption of the beclin 1 autophagy gene. J Clin Invest 112, 1809-1820 
11. Yue Z, Jin S, Yang C, Levine AJ and Heintz N (2003) Beclin 1, an autophagy gene essential for early embryonic development, is a haploinsufficient tumor suppressor. Proc Natl Acad Sci U S A 100, 15077-15082

12. Komatsu M, Waguri S, Ueno T et al (2005) Impairment of starvation-induced and constitutive autophagy in Atg7-deficient mice. J Cell Biol 169, 425-434

13. Hara T, Nakamura K, Matsui M et al (2006) Suppression of basal autophagy in neural cells causes neurodegenerative disease in mice. Nature 441, 885-889

14. Kuma A, Hatano M, Matsui $M$ et al (2004) The role of autophagy during the early neonatal starvation period. Nature 432, 1032-1036

15. Qu X, Zou Z, Sun Q et al (2007) Autophagy genedependent clearance of apoptotic cells during embryonic development. Cell 128, 931-946

16. O'Prey J, Sakamaki J, Baudot A et al (2017) Application of CRISPR/Cas9 to Autophagy Research. Methods Enzymol 588, 79-108

17. Ishino $Y$, Shinagawa $H$, Makino $K$, Amemura $M$ and Nakata A (1987) Nucleotide sequence of the iap gene, responsible for alkaline phosphatase isozyme conversion in Escherichia coli, and identification of the gene product. J Bacteriol 169, 5429-5433

18. Barrangou R and van der Oost J (2012) CRISPR-Cas systems: RNA-mediated adaptive immunity in bacteria and archaea. Springer Science \& Business Media 1-31

19. Mojica FJ, Diez-Villasenor C, Garcia-Martinez J and Soria E (2005) Intervening sequences of regularly spaced prokaryotic repeats derive from foreign genetic elements. J Mol Evol 60, 174-182

20. Garneau JE, Dupuis ME, Villion M et al (2010) The CRISPR/Cas bacterial immune system cleaves bacteriophage and plasmid DNA. Nature 468, 67-71

21. Deltcheva E, Chylinski K, Sharma CM et al (2011) CRISPR RNA maturation by trans-encoded small RNA and host factor RNase III. Nature 471, 602-607

22. Gasiunas G, Barrangou R, Horvath $P$ and Siksnys $V$ (2012) Cas9-crRNA ribonucleoprotein complex mediates specific DNA cleavage for adaptive immunity in bacteria. Proc Natl Acad Sci U S A 109, E2579-2586

23. Jinek M, Chylinski K, Fonfara I, Hauer M, Doudna JA and Charpentier E (2012) A programmable dual-RNAguided DNA endonuclease in adaptive bacterial immunity. Science 337, 816-821

24. Xie S, Shen B, Zhang C, Huang X and Zhang Y (2014) sgRNAcas9: a software package for designing CRISPR sgRNA and evaluating potential off-target cleavage sites. PLoS One 9, e100448

25. Horvath $P$ and Barrangou R (2010) CRISPR/Cas, the immune system of bacteria and archaea. Science 327, $167-170$

26. Marraffini LA and Sontheimer EJ (2008) CRISPR interference limits horizontal gene transfer in staphylococci by targeting DNA. Science 322, 1843-1845

27. Hale CR, Zhao P, Olson S et al (2009) RNA-guided RNA cleavage by a CRISPR RNA-Cas protein complex. Cell $139,945-956$

28. Barrangou R and Marraffini LA (2014) CRISPR-Cas systems: Prokaryotes upgrade to adaptive immunity. Mol
Cell 54, 234-244

29. Ran FA, Hsu PD, Wright J, Agarwala V, Scott DA and Zhang F (2013) Genome engineering using the CRISPRCas9 system. Nat Protoc 8, 2281-2308

30. Jinek M, Jiang F, Taylor DW et al (2014) Structures of Cas9 endonucleases reveal RNA-mediated conformational activation. Science 343, 1247997

31. Nishimasu H, Ran FA, Hsu PD et al (2014) Crystal structure of Cas9 in complex with guide RNA and target DNA. Cell 156, 935-949

32. Hsu PD, Lander ES and Zhang F (2014) Development and applications of CRISPR-Cas9 for genome engineering. Cell 157, 1262-1278

33. Jiang F and Doudna JA (2017) CRISPR-Cas9 Structures and Mechanisms. Ann Rev Biophys 46, 1

34. Shen B, Brown KM, Lee TD and Sibley LD (2014) Efficient gene disruption in diverse strains of Toxoplasma gondii using CRISPR/CAS9. MBio 5, e01114-01114

35. Sternberg $\mathrm{SH}$, Redding $\mathrm{S}$, Jinek $\mathrm{M}$, Greene EC and Doudna JA (2014) DNA interrogation by the CRISPR RNA-guided endonuclease Cas9. Nature 507, 62-67

36. Kleinstiver BP, Prew MS, Tsai SQ et al (2015) Engineered CRISPR-Cas9 nucleases with altered PAM specificities. Nature 523, 481-485

37. Yamano T, Nishimasu H, Zetsche B et al (2016) Crystal Structure of Cpf1 in Complex with Guide RNA and Target DNA. Cell 165, 949-962

38. Makarova KS, Wolf Yl, Alkhnbashi OS et al (2015) An updated evolutionary classification of CRISPR-Cas systems. Nat Rev Microbiol 13, 722-736

39. Jiang W, Bikard D, Cox D, Zhang F and Marraffini LA (2013) RNA-guided editing of bacterial genomes using CRISPR-Cas systems. Nat Biotechnol 31, 233-239

40. Cong L, Ran FA, Cox D et al (2013) Multiplex genome engineering using CRISPR/Cas systems. Science 339, 819-823

41. Xu T, Li Y, Van Nostrand JD, He Z and Zhou J (2014) Cas9-based tools for targeted genome editing and transcriptional control. Appl Environ Microbiol 80, 1544-1552

42. Wang $H$, Yang $H$, Shivalila CS et al (2013) One-step generation of mice carrying mutations in multiple genes by CRISPR/Cas-mediated genome engineering. Cell 153, 910-918

43. Ran FA, Hsu PD, Lin CY et al (2013) Double nicking by RNA-guided CRISPR Cas9 for enhanced genome editing specificity. Cell 154, 1380-1389

44. Qi LS, Larson MH, Gilbert LA et al (2013) Repurposing CRISPR as an RNA-guided platform for sequence-specific control of gene expression. Cell 152, 1173-1183

45. Schwank G, Koo BK, Sasselli V et al (2013) Functional repair of CFTR by CRISPR/Cas9 in intestinal stem cell organoids of cystic fibrosis patients. Cell Stem Cell 13, 653-658

46. Smith C, Gore A, Yan W et al (2014) Whole-genome sequencing analysis reveals high specificity of CRISPR/ Cas9 and TALEN-based genome editing in human iPSCs. Cell Stem Cell 15, 12-13

47. Ousterout DG, Kabadi AM, Thakore PI, Majoros WH and Reddy TE (2015) Multiplex CRISPR/Cas9-based 
genome editing for correction of dystrophin mutations that cause Duchenne muscular dystrophy. Nat Commun 6,6244

48. Pan Y, Xiao L, Li AS et al (2013) Biological and biomedical applications of engineered nucleases. Mol Biotechnol 55, 54-62

49. Liu R, Paxton WA, Choe $S$ et al (1996) Homozygous defect in HIV-1 coreceptor accounts for resistance of some multiply-exposed individuals to HIV-1 infection. Cell 86, 367-377

50. Lombardo A, Genovese P, Beausejour CM et al (2007) Gene editing in human stem cells using zinc finger nucleases and integrase-defective lentiviral vector delivery. Nat Biotechnol 25, 1298-1306

51. Musunuru K, Pirruccello JP, Do R et al (2010) Exome sequencing, ANGPTL3 mutations, and familial combined hypolipidemia. N Engl J Med 363, 2220-2227

52. Ding Q, Regan SN, Xia Y, Oostrom LA, Cowan CA and Musunuru K (2013) Enhanced efficiency of human pluripotent stem cell genome editing through replacing TALENs with CRISPRs. Cell Stem Cell 12, 393-394

53. Fu Y, Foden JA, Khayter C et al (2013) High-frequency off-target mutagenesis induced by CRISPR-Cas nucleases in human cells. Nat Biotechnol 31, 822-826

54. Sander JD and Joung JK (2014) CRISPR-Cas systems for editing, regulating and targeting genomes. Nat Biotechnol 32, 347-355

55. Mout R, Ray M, Yesilbag Tonga G et al (2017) Direct Cytosolic Delivery of CRISPR/Cas9-Ribonucleoprotein for Efficient Gene Editing. ACS Nano 11, 2452-2458

56. Jiang C, Mei M, Li B et al (2017) A non-viral CRISPR/ Cas9 delivery system for therapeutic gene targeting in vivo. Cell Res 27, 440-443

57. Cho SW, Lee J, Carroll D, Kim JS and Lee J (2013) Heritable gene knockout in Caenorhabditis elegans by direct injection of Cas9-sgRNA ribonucleoproteins. Genetics 195, 1177-1180

58. Jiang $W$, Zhou $\mathrm{H}$, Bi H, Fromm M, Yang B and Weeks DP (2013) Demonstration of CRISPR/Cas9/sgRNA-mediated targeted gene modification in Arabidopsis, tobacco, sorghum and rice. Nucleic Acids Res 41, e188

59. Jackson AL and Linsley PS (2010) Recognizing and avoiding siRNA off-target effects for target identification and therapeutic application. Nat Rev Drug Discov 9, 57-67

60. Hsu PD, Scott DA, Weinstein JA et al (2013) DNA targeting specificity of RNA-guided Cas9 nucleases. Nat Biotechnol 31, 827-832

61. Slaymaker IM, Gao L, Zetsche B, Scott DA, Yan WX and Zhang F (2016) Rationally engineered Cas9 nucleases with improved specificity. Science 351, 84-88

62. Kleinstiver BP, Pattanayak V, Prew MS et al (2016) High-fidelity CRISPR-Cas9 nucleases with no detectable genome-wide off-target effects. Nature 529, 490-495

63. Wu YT, Tan HL, Shui G et al (2010) Dual Role of 3-Methyladenine in Modulation of Autophagy via Different Temporal Patterns of Inhibition on Class I and III Phosphoinositide 3-Kinase. J Biol Chem 285, 10850-10861

64. Yang YP, Hu LF, Zheng HF et al (2013) Application and interpretation of current autophagy inhibitors and activators. Acta Pharmacol Sin 34, 625-635

65. Yuan N, Song L, Zhang S et al (2015) Bafilomycin A1 targets both autophagy and apoptosis pathways in pediatric B-cell acute lymphoblastic leukemia. Haematologica 100, 345-356

66. Maycotte P, Aryal S, Cummings CT, Thorburn J, Morgan MJ and Thorburn A (2012) Chloroquine sensitizes breast cancer cells to chemotherapy independent of autophagy. Autophagy 8, 200-212

67. Liu Y, Lin J, Zhang M et al (2016) PINK1 is required for timely cell-type specific mitochondrial clearance during Drosophila midgut metamorphosis. Dev Biol 419, 357-372

68. Sasaki T, Lian S, Khan A et al (2017) Autolysosome biogenesis and developmental senescence are regulated by both Spns1 and v-ATPase. Autophagy 13, 386-403

69. Padman BS, Nguyen TN and Lazarou M (2017) Autophagosome formation and cargo sequestration in the absence of LC3/GABARAPs. Autophagy 13, 1-3

70. Horne DJ, Graustein AD, Shah JA et al (2016) Human ULK1 Variation and Susceptibility to Mycobacterium tuberculosis Infection. J Infect Dis 214, 1260-1267

71. Wang J, Fang Y, Yan L et al (2016) Erythroleukemia cells acquire an alternative mitophagy capability. Sci Rep 6, 24641

72. Kim NY, Han BI and Lee M (2016) Cytoprotective role of autophagy against $\mathrm{BH} 3$ mimetic gossypol in ATG5 knockout cells generated by CRISPR-Cas9 endonuclease. Cancer Lett 370, 19-26

73. Cao Y, Cai J, Li X, Yuan N and Zhang S (2016) Autophagy governs erythroid differentiation both in vitro and in vivo. Hematology 21, 225-233

74. Ohshima J, Lee Y, Sasai M et al (2014) Role of mouse and human autophagy proteins in IFN-gamma-induced cell-autonomous responses against Toxoplasma gondii. J Immunol 192, 3328-3335

75. Dejesus R, Moretti F, McAllister G et al (2016) Functional CRISPR screening identifies the ufmylation pathway as a regulator of SQSTM1/p62. 5. Elife e17290

76. Hatoum-Aslan A, Maniv I, Samai P and Marraffini LA (2014) Genetic Characterization of Antiplasmid Immunity through a Type III-A CRISPR-Cas System. J Bacteriol 196, 310-317

77. Mali P, Aach J, Stranges PB et al (2013) CAS9 transcriptional activators for target specificity screening and paired nickases for cooperative genome engineering. Nat Biotechnol 31, 833-838

78. Cho SW, Kim S, Kim JM and Kim J-S (2013) Targeted genome engineering in human cells with the Cas9 RNA-guided endonuclease. Nat Biotechnol 31, 230-232

79. Chang N, Sun C, Gao L et al (2013) Genome editing with RNA-guided Cas9 nuclease in zebrafish embryos. Cell Res 23, 465-472

80. Miao J, Guo D, Zhang J et al (2013) Targeted mutagenesis in rice using CRISPR-Cas system. Cell Res 23, 1233

81. Jiang $\mathrm{W}$, Maniv I, Arain F, Wang $\mathrm{Y}$, Levin $\mathrm{BR}$ and Marraffini LA (2013) Dealing with the Evolutionary Downside of CRISPR Immunity: Bacteria and Beneficial 
Plasmids. PLoS Genet 9, e1003844

82. Xie K and Yang Y (2013) RNA-guided genome editing in plants using a CRISPR-Cas system. Mol Plant 6, 1975-1983

83. Shan Q, Wang Y, Li J et al (2013) Targeted genome modification of crop plants using a CRISPR-Cas system. Nat Biotechnol 31, 686-688

84. Fodor E, Sigmond T, Ari E et al (2017) Methods to Study Autophagy in Zebrafish. Methods Enzymol 588, 467-496

85. Gratz SJ, Cummings AM, Nguyen JN et al (2013) Genome engineering of Drosophila with the CRISPR RNA-guided Cas9 nuclease. Genetics 194, 1029-1035

86. Friedland $\mathrm{AE}$, Tzur $\mathrm{YB}$, Esvelt $\mathrm{KM}$, Colaiácovo MP, Church GM and Calarco JA (2013) Heritable genome editing in C. elegans via a CRISPR-Cas9 system. Nat Methods 10, 741-743

87. Waaijers S, Portegijs V, Kerver J et al (2013) CRISPR/ Cas9-Targeted Mutagenesis in Caenorhabditis elegans. Genetics 195, 1187-1191

88. Nakayama $T$, Fish MB, Fisher $M$, Oomen-Hajagos J, Thomsen GH and Grainger RM (2013) Simple and efficient CRISPR/Cas9-mediated targeted mutagenesis in Xenopus tropicalis. Genesis 51, 835-843

89. DiCarlo JE, Norville JE, Mali P, Rios X, Aach J and Church GM (2013) Genome engineering in Saccharomyces cerevisiae using CRISPR-Cas systems. Nucleic Acids Res 41, 4336-4343

90. Li J-F, Norville JE, Aach J et al (2013) Multiplex and homologous recombination-mediated genome editing in Arabidopsis and Nicotiana benthamiana using guide RNA and Cas9. Nat Biotechnol 31, 688-691

91. Nekrasov V, Staskawicz B, Weigel D, Jones JD and Kamoun S (2013) Targeted mutagenesis in the model plant Nicotiana benthamiana using Cas9 RNA-guided endonuclease. Nat Biotechnol 31, 691-693

92. Dickinson DJ, Ward JD, Reiner DJ and Goldstein B (2013) Engineering the Caenorhabditis elegans genome using Cas9-triggered homologous recombination. Nat Methods 10, 1028-1034

93. Bikard D, Jiang W, Samai P, Hochschild A, Zhang F and Marraffini LA (2013) Programmable repression and activation of bacterial gene expression using an engineered CRISPR-Cas system. Nucleic Acids Res 41, 7429-7437

94. Gilbert LA, Larson MH, Morsut L et al (2013) CRISPRmediated modular RNA-guided regulation of transcription in eukaryotes. Cell 154, 442-451

95. Suzuki $H$, Kaizuka T, Mizushima $N$ and Noda NN (2015) Structure of the Atg101-Atg13 complex reveals essential roles of Atg101 in autophagy initiation. Nat Struct Mol Biol 22, 572-580

96. Tang $\mathrm{Y}, \mathrm{Li} \mathrm{J}$, Li F et al (2015) Autophagy protects intestinal epithelial Cells against Deoxynivalenol toxicity by alleviating oxidative stress via IKK signaling pathway. Free Radi Biol Med 89, 944-951

97. Allavena G, Boyd C, Oo KS, Maellaro E, Zhivotovsky B and Kaminskyy VO (2016) Suppressed translation and ULK1 degradation as potential mechanisms of autophagy limitation under prolonged starvation. Autophagy 12 2085-2097

98. Kaizuka T and Mizushima N (2016) Atg13 is essential for autophagy and cardiac development in mice. Mol Cell Biol 36, 585-595

99. Tsuboyama K, Koyama-Honda I, Sakamaki Y, Koike M, Morishita H and Mizushima N (2016) The ATC conjugation systems are important for degradation of the inner autophagosomal membrane. Science 354, 10361041

100. Stavoe AK, Hill SE, Hall DH and Colon-Ramos DA (2016) KIF1A/UNC-104 Transports ATG-9 to Regulate Neurodevelopment and Autophagy at Synapses. Dev Cell 38, 171-185

101. Fulcher LJ, Macartney T, Bozatzi P, Hornberger A Rojas-Fernandez A and Sapkota GP (2016) An affinitydirected protein missile system for targeted proteolysis. Open Biol 6, 160255

102. Tan X, Thapa N, Liao Y, Choi S and Anderson RA (2016) Ptdlns $(4,5)$ P2 signaling regulates ATG14 and autophagy. Proc Natl Acad Sci U S A 113, 10896-10901

103. Corcelle-Termeau $E$, Vindeløv SD, Hämälistö $S$ et al (2016) Excess sphingomyelin disturbs ATG9A trafficking and autophagosome closure. Autophagy 12, 833-849 